\title{
¿Por qué un Máster en Psicología Educativa?
}

\section{Why a Master's in Educational Psychology?}

\author{
María Pérez Solís \\ Universidad Complutense de Madrid, España
}

\begin{abstract}
Resumen. La Psicología ha experimentado en las últimas décadas un importante desarrollo y una creciente implantación en diversos ámbitos de actuación profesional, entre ellos el educativo. En España, no obstante, la Orientación Educativa ha sufrido un lamentable retroceso, favorecido entre otros factores por la seudo-profesionalización. El objeto de este artículo es poner de manifiesto la necesidad de una formación especializada que corrija el desajuste existente entre la oferta de formación y las capacidades y competencias profesionales que la sociedad requiere del psicólogo educativo. En concreto, se presenta una propuesta en la que se defiende la necesidad de un Máster específico en Psicología Educativa, frente al existente de Formación del Profesorado, en la especialidad de Orientación Educativa. Palabras clave: máster en Psicología Educativa, cualificación profesional, profesor orientador, seudo-profesional.
\end{abstract}

\begin{abstract}
Psychology has experienced significant development in recent years, as well as achieving an increased presence in various fields of professional activity, including education. In Spain, however, progress in Educational Guidance has been unfortunately slow. This has been due to, among other factors, its pseudo-professionalized state. The purpose of this article is to highlight the need for specialized training to correct the imbalance between the training currently received by these workers and the professional skills and competences which are required today of the educational psychologist. Specifically, it presents a proposal that supports the need for a specific master's degree in Educational Psychology, rather than existing Teacher Training specializing in Educational Guidance. Keywords: master's in Educational Psychology, professional qualification, guidance teacher, pseudo-professional.
\end{abstract}

\section{Aspectos introductorios}

En la actualidad, las enseñanzas universitarias españolas están organizadas en dos ciclos que se corresponden con tres niveles de cualificación universitaria: grado, máster y doctor, en sintonía con el marco europeo de cualificaciones para la educación superior, basado en los denominados descriptores de Dublín y de acuerdo con las reglas que establece la

La correspondencia sobre este artículo se puede dirigirse a la autora al Servicio de Orientación de la Facultad de Psicología, Universidad Complutense de Madrid Campus de Somosaguas s/n 28223 Madrid. Email:mperezso@psi.ucm.es
Directiva de Reconocimiento de Cualificaciones Profesionales (Directiva 2005/36/CE) para el reconocimiento mutuo de cualificaciones profesionales entre los Estados miembros. Esta normativa afecta a diversas profesiones reguladas, que únicamente pueden ser desempeñadas en el caso de haber adquirido determinadas cualificaciones profesionales, entre las que se incluye la Psicología. No obstante, la citada Directiva, al parecer no permite alcanzar uno de sus objetivos fundamentales, como es el de facilitar la movilidad de los profesionales dentro del espacio europeo. Por ello, y tras la evaluación realizada por la Comisión Europea durante el pasado año, se ha 
iniciado recientemente un periodo de consulta (que finalizó el 15 de marzo), a los Ministros y responsables de las distintas áreas de profesiones reguladas, colegios profesionales, asociaciones, profesionales y consumidores, previo a la elaboración del informe definitivo de la Comisión Europea para la modificación de la actual Directiva prevista para 2012.

La reforma de los planes de estudio de Psicología en los distintos países de Europa contempla un modelo de grado de tres o cuatro años, no especializado, que no capacita para el ejercicio de la profesión y un postgrado con diversos másteres específicos (al menos en Psicología Educativa, Psicología Clínica y Psicología del Trabajo), que tienen como finalidad la adquisición de una formación avanzada, orientada a la especialización académica o profesional, o bien a promover la iniciación en tareas investigadoras. En países de nuestro entorno, como Alemania, Reino Unido, Francia, Irlanda o Grecia, la competencia para el ejercicio profesional en distintos campos de la Psicología la otorga el máster, que se cursará si previamente se ha obtenido el grado en Psicología. En algunos países, como Italia, se requiere además un año de prácticas supervisadas y un examen estatal.

Asimismo, en la mayoría de los países europeos, existe la diferenciación entre el título y la cualificación. Además, suelen existir agencias de habilitación profesional que se encargan de dotar a los recién egresados de la acreditación necesaria para el desempeño de sus tareas profesionales. En España, por el contrario, no existe distinción entre las habilidades profesionales que comportan un título académico y las atribuciones profesionales que el Estado confiere para el desempeño profesional. Precisamente, la actual normativa española atribuye al título oficial de grado un doble efecto académico y profesional, en virtud del cual su poseedor goza de la plenitud de derechos académicos a él inherentes, al tiempo que le capacita plenamente para el desempeño profesional. Por tanto, el título oficial español es, en la mayoría de los casos, académico y profesional, sin que ello suponga la no existencia en nuestro ordenamiento de títulos específicamente profesiona- les y no académicos, como los correspondientes a las especialidades médicas y arquitectura o para aquellas profesiones como la actividad docente en la enseñanza secundaria, que para ejercer se requiere, además del título académico, la posesión del título profesional de especialización didáctica.

Entre las especialidades de Enseñanza Secundaria, se encuentra la de Orientación Educativa, denominación actual de la especialidad de Psicología y Pedagogía, según contempla la disposición adicional cuarta del Real Decreto 1834/2008, de 8 de noviembre (BOE de 28 de noviembre de 2008). Esta especialidad, existente en el Cuerpo de Enseñanza Secundaria desde 1991, no puede convertirse en una especialización en "Orientación Educativa" para profesionales de ámbitos distintos de la Psicología y de la Pedagogía. La existencia de esta especialidad, en ningún caso ha tenido su razón de existir en función de los aspectos docentes, inherentes a las otras especialidades (Pérez Solís, 2010). Lamentablemente, la especialidad de Orientación Educativa, dentro del Máster de Formación del Profesorado, parece que se ha convertido en el medio para formar seudo-profesionales, con el coste social que ello conlleva, dado que funciones como la evaluación diagnóstica de la discapacidad la podrá realizar cualquier docente que haya cursado la citada especialidad (Real Decreto 860/2010, de 2 de julio, BOE de 17 de julio de 2010). La invertebración de la formación inicial afecta profundamente a su profesionalización y el no tenerlo en cuenta, no sólo va en detrimento de un servicio especializado sino de la propia calidad educativa.

\section{Justificación}

\section{Interés y relevancia científica, académica $y$ profesional}

Llegado a este punto, cabe preguntarse por la necesidad o no de un máster específico en Psicología Educativa, para aquellos psicólogos que deseen desarrollar su actividad en contextos educativos (a 
partir de ahora psicólogos educativos). Evidentemente, la respuesta es rotundamente sí, y así se recoge, en el acuerdo firmado el 21 de septiembre de 2010 en la Facultad de Psicología de la Universidad Complutense de Madrid, por la Conferencia de Decanos de Psicología (CDPUE), Consejo General de Colegios Oficiales de Psicólogos, la Federación de Asociaciones de Directivos de Centros Educativos Públicos (FEDADI), la Confederación Española de Asociaciones de Madres y Padres de Alumnos (CEAPA) y la Confederación Católica Nacional de Padres de Familia y Padres de Alumnos (CONCAPA). Es decir, formadores, profesionales y usuarios, ponen de manifiesto ante la opinión pública, que las necesidades que tiene la Comunidad Educativa sólo pueden ser adecuadamente satisfechas por profesionales cualificados. El citado acuerdo, indica en su apartado 4 que: "El psicólogo, graduado o licenciado en Psicología, ha de haber cursado además un máster específico de Psicología de la Educación, centrado en la capacitación profesional para ejercer en contextos educativos, dado que el actual máster de Formación del Profesorado no es, en modo alguno, el medio adecuado para formar a estos profesionales".

Compartimos plenamente este acuerdo y como de forma unánime ha manifestado la Conferencia de Decanos, estimamos necesario desarrollar una formación especializada, enfocada al ejercicio profesional pleno, más allá de la titulación de Grado. Todo psicólogo, debe tener una formación básica común, y luego una formación especializada atendiendo al ámbito educativo, clínico, social y laboral en el que va a ejercer. Evidentemente, el máster que proponemos de Psicología Educativa, sustituiría al citado máster de Formación del Profesorado para los psicólogos educativos.

En esta línea y en aras de una mayor autonomía universitaria, hemos pasado hoy día de tener un catálogo a un registro de títulos oficiales y de unas directrices generales comunes para los planes de estudio a sólo unas directrices mínimas comunes en cuanto a requisitos de acceso, duración y organización. Desde este nuevo modelo normativo corremos el riesgo de que se aprueben titulaciones que no garanticen la adecuada cualificación profesional. En este sentido, resulta más que preocupante la proliferación de másteres que con el título de Psicología Educativa, cursan alumnos de distintos ámbitos científicos, careciendo de una sólida formación inicial en Psicología.

La especialización, en consecuencia, se ha de apoyar en una sólida formación inicial fundamental y básica acorde en su contenido con su ámbito científico o profesional, como parece contemplar el Real Decreto 861/2010, de 2 de julio, por el que se modifica el Real Decreto 1393/2007, de 29 de octubre, de ordenación de las enseñanzas universitarias oficiales, que en su artículo único, cuatro, 3 dice:

"Los títulos oficiales de Máster Universitario podrán incorporar especialidades en la programación de sus enseñanzas que se correspondan con su ámbito científico, humanístico, tecnológico o profesional. En todo caso, las Administraciones Públicas velarán por que la denominación del título sea acorde con su contenido y en su caso, con la normativa específica de aplicación, y no conduzca a error sobre su nivel o efectos académicos ni a confusión sobre su contenido y, en su caso, efectos profesionales".

También indica en el apartado cinco, 9. "Cuando se trate de títulos que habiliten para el ejercicio de actividades profesionales reguladas en España, el Gobierno establecerá las condiciones a las que deberán adecuarse los correspondientes planes de estudios, que además deberán ajustarse, en su caso, a la normativa europea aplicable”.

En síntesis, el rol del psicólogo educativo, no debe ser desempeñado por cualquier docente que curse el citado master de Formación del Profesorado, como así parece entenderlo el MEC cuando interpreta, a nuestro juicio de forma errónea, lo que desde el ámbito de la ciencia y de la excelencia se entiende por "servicios profesionales especializados" y nos inquieta que desde la Administración Educativa se vaya en otra dirección y se favorezca la generalización de roles frente a la especialización y capacitación, como se pone de manifiesto en la 
reciente Orden EDU/849/2010 (BOE de 18 de marzo), por la que se regula la ordenación de la educación del alumnado con necesidad de apoyo educativo y los servicios de orientación educativa en su ámbito de gestión. Es preocupante en suma, en especial para aquellos padres que tienen hijos discapacitados, que funciones específicas como la valoración diagnóstica, asesoramiento psicológico o la propuesta de intervención ajustada a las necesidades educativas especiales que presentan, las lleven a cabo seudo-profesionales.

Finalmente, apoyamos plenamente lo que recientemente se plantea desde la Asociación Internacional de Psicología Escolar (ISPA), nos referimos a establecer unas directrices internacionales en materia de formación de los psicólogos educativos, en las que se reflejen unas metas formativas comunes a todos los países del mundo. De este modo, alcanzaríamos no sólo un importante éxito frente al intrusismo profesional y la endogamia de determinados departamentos universitarios españoles, bien situados cerca del poder, sino también la mejora de la calidad en los planes de formación y en la práctica profesional.

\section{Antecedentes y situación profesional del psicólogo educativo}

Con el actual ordenamiento normativo, hemos retrocedido a épocas pasadas, en lo que a prestación de servicios especializados se refiere. Efectivamente, funciones como el asesoramiento psicológico lo puede hacer, según el MEC, cualquier profesor especialista en orientación educativa. No obstante, debe recordarse que desde sus orígenes (Ley 14/1970 de 4 de agosto, en sus art. 9, 125 y 127) y en el marco de la Ley 13/1982, de 7 de abril, sobre Integración Social de los Minusválidos, se estableció que "la Orientación Educativa" debía ser ofrecida en el marco de un grupo de servicios especializados con funciones preventivas, compensatorias y de soporte técnico y en las distintas etapas educativas no universitarias. Con la Orden del 30 de Abril de 1977 se crean las primeras plazas no escalafonadas de psicólogos, de entre el funcionariado, para atender la EGB y la Educación Especial. También a través la O.M. de 9 de Septiembre de 1982, en el marco de la Ley de Integración de los Minusválidos del 7 de abril de 1982, se regula el acceso de estos profesionales como contratados laborales para el ámbito de la Educación Especial.

Con posterioridad, a raíz de la huelga de estudiantes de Enseñanza Media del curso 1986/1987 y en respuesta a sus peticiones, surgen con carácter experimental los Proyectos de Orientación Educativa y Profesional (O.M.de 4 de Junio de 1987, O.M.de 25 de Febrero de 1988 y O.M.de 28 de Marzo de 1989), pero en ellos la función orientadora la podía ejercer cualquier profesor sin que necesariamente fuera psicólogo o pedagogo, lo que llevó esta iniciativa al fracaso. A partir del Real Decreto 929 de 18 de Junio de 1993, la orientación en Enseñanza Secundaria corre a cargo de los Departamentos de Orientación, en los que al menos uno de sus miembros debe ser especialista en Psicología o en Pedagogía. En esta misma línea, la Ley 1/1990 de 3 de octubre, en la Disposición Adicional $3^{\mathrm{a}} \mathrm{e}$, como la actual Ley Orgánica 2/2006, de 3 de mayo de Educación, establecen la creación de servicios especializados de orientación educativa, psicopedagógica y profesional (Pérez Solís, 2010).

Es evidente que la necesidad del apoyo técnico del especialista en Psicología se contempla en un extenso marco normativo: Real Decreto 334/1985, de 6 de marzo, de ordenación de la Educación Especial; Real Decreto 696/1995, de 28 de abril, de la ordenación de los alumnos con necesidades educativas especiales; Real Decreto 222/1996, de 28 de febrero, de ordenación de las acciones dirigidas a la compensación de desigualdades en educación, etc. Asimismo, las funciones y estructura de estos servicios están reguladas por O.M. de 9 de diciembre de 1992 y normativas sucesivas como la Orden del 14 de febrero de 1996 y la Resolución de 29 de abril de 1996.

A raíz de la aprobación de los Reales Decretos $55 / 2005$ y $56 / 2005$ y el posterior $1393 / 2007$, el 
Máster de Formación del Profesorado es uno de los títulos oficiales que habilita para el ejercicio de profesiones reguladas en España. Con posterioridad, en el Real Decreto 1834/2008 (BOE 28 de noviembre de 2008), se definen las condiciones de formación para el ejercicio de la docencia en la educación secundaria obligatoria, el bachillerato, la formación profesional y con ello, se produce un cambio sustancial en el concepto de especialización y cualificación profesional.

La base jurídica de la acreditación de las cualificaciones profesionales a través de las ofertas formativas del sistema educativo y del laboral es, respectivamente, la citada Ley Orgánica 2/2006, de 3 de mayo y su desarrollo en los certificados de profesionalidad en el Real Decreto 1834/2008, de 8 de noviembre en el que en la disposición final primera reserva al Estado la competencia para establecer las bases del régimen jurídico de las Administraciones Públicas y la competencia de regulación de las condiciones de obtención, expedición y homologación de títulos académicos y profesionales y la competencia para establecer normas básicas para el desarrollo del artículo 27 de la Constitución.

La Ley Orgánica 4/2007, de 12 de abril, por la que se modifica la Ley Orgánica 6/2001, de 21 de diciembre, de Universidades, sienta las bases para realizar una profunda modernización de la Universidad española, proponiéndose entre sus objetivos fundamentales una nueva organización de las enseñanzas que permita fomentar la movilidad de los estudiantes, tanto a nivel interno como dentro de Europa y a nivel internacional. No obstante, con el desarrollo del actual marco normativo, Real Decreto1393/2007 de 29 de octubre, el Real Decreto $1834 / 2008$ de 8 de noviembre, Real Decreto 860/2010, de 2 de julio y la Orden EDU/849/2010, no será posible, dado que las condiciones de formación para ejercer las funciones de Orientación Educativa como establece el artículo 5, 2 del citado Real Decreto 860/2010, de 2 de julio, nos aleja de los estándares europeos de cualificación y favorece la seudo-profesionalización.

Finalmente, consideramos que la regulación del régimen de acceso a la profesión de psicólogo educativo es una exigencia derivada de las previsiones constitucionales y la interpretación que el Tribunal Constitucional ha efectuado principalmente de los arts. 35, 36 y 149.1.30 a de la Constitución Española (CE), por la que serán profesiones reguladas aquellas actividades profesionales en cuyo desarrollo puedan verse implicados intereses públicos o generales y en las que exista una relación determinante entre la titulación exigida y la especificidad de la actividad a realizar, determinadas por la intervención del Legislador.

\section{El psicólogo educativo en el contexto internacional}

En el ámbito internacional, el psicólogo educativo cuenta con un amplio reconocimiento académico y profesional. La Asociación Americana de Psicología (APA), referente internacional en el campo de la Psicología, tiene entre sus divisiones, dos que reconocen este campo de especialización. Concretamente la división 15: "Educational Psychology" y la 16: "School Psychology". En este ámbito, se encuentran entre otras, la Asociación Internacional de Psicología Escolar (ISPA) y la European Association for Research on Learning and Instruction (EARLI).

Los compromisos adquiridos por el Gobierno, al suscribir la Declaración de Bolonia en 1999 y los posteriores acuerdos de los Ministros responsables de Educación Superior de la Unión Europea, de Praga (2001), Berlín (2003), Bergen (2005), Londres (2007), Lovaina (2009) y Budapest-Viena (2010), han supuesto una trascendental transformación del sistema universitario español. Asimismo, los objetivos del Proceso de Copenhague para la cooperación reforzada en la formación y las enseñanzas profesionales y el reciente ejercicio de la OCDE sobre reconocimiento de "learning outcomes" (OECD 2010), que relaciona el reconocimiento de las cualificaciones en el contexto de sus beneficios económicos, educativos, sociales y persona- 
les, obliga a que las especializaciones se apoyen en una sólida formación inicial y básica, acorde en su contenido con su ámbito científico o profesional.

El Consejo de Europa y el Parlamento Europeo, aprobaron la Directive on the recognition of professional qualifications (European Union, June 6, 2005) que en su artículo 15 establece la posibilidad de que organizaciones profesionales europeas representativas realicen propuestas de "plataformas comunes" o requerimientos mínimos que se deberían cumplir para el reconocimiento automático, entre los países de la Unión Europea (UE), de las cualificaciones profesionales sin medidas compensatorias añadidas.

De acuerdo con esta directiva, la Federación Europea de Asociaciones de Psicólogos (EFPA), considera que para el ejercicio profesional de la Psicología se requiere de una formación mínima de 6 años (grado, máster específico y prácticas supervisadas). En esta misma línea, se sitúa el acuerdo al que hicimos referencia con anterioridad, en la que todos, formadores, profesionales y usuarios manifiestan, que las necesidades de la Comunidad Educativa, sólo pueden ser adecuadamente satisfechas por profesionales cualificados. También iniciativas como el Proyecto ESPIL, aprobado por la Unión Europea a finales del 2009, incluyen recomendaciones sobre el desarrollo de futuros perfiles profesionales y sobre las competencias requeridas a los psicólogos que trabajan en el sistema educativo en Europa.

A nivel europeo, cada vez con más frecuencia se relaciona el éxito o el fracaso escolar con la existencia o no de servicios de apoyo psicoeducativo. En la reunión en Bratislava (2009) del Network of Psychologists in the Educational System (NEPES), grupo de trabajo de la red de psicólogos educativos de la European Federation of Psychologists, Associations (EFPA), se pone de manifiesto la promoción de los servicios psicológicos que algunos de los países más avanzados estaban llevando a cabo en sus sistemas educativos, como por ejemplo Suecia que ha introducido en su nueva ley educativa las competencias específicas del psicólogo en el siste- ma escolar. También Alemania y Dinamarca presentan avances similares, incorporando a los psicólogos con perfiles definidos en distintos niveles dentro de sus sistemas educativos.

En España aún se ha de superar el importante desajuste existente entre la oferta de formación y las capacidades y competencias profesionales que la sociedad requiere de los egresados universitarios. En este sentido, urge disponer de un referente de cualificaciones profesionales en el ámbito de la enseñanza superior adaptadas al nuevo marco europeo, desarrollando a tal fin el Marco Español de Cualificaciones para la Educación Superior (MECES).

\section{Objetivos y competencias}

La Psicología ha experimentado en las últimas décadas un importante desarrollo y una creciente implantación en diversos ámbitos de actuación profesional, por lo que el título de grado de carácter generalista no puede satisfacer plenamente la enorme diversificación de las funciones que ejercen los psicólogos. Las enseñanzas de máster tienen como finalidad completar esa formación inicial de grado mediante una formación avanzada, orientada a la especialización académica, profesional o investigadora. El propósito de estas enseñanzas en Psicología, será el poder aplicar en la práctica profesional los principios psicológicos, conocimientos, modelos y métodos científicos con el fin de promover el desarrollo, bienestar y eficacia de los individuos, grupos, organizaciones y sociedad.

Este trabajo se circunscribe al perfil del psicólogo educativo y exclusivamente a su vertiente profesional, sin menosprecio de otros tipos de másteres como el académico e investigador. En consecuencia, el objetivo general del máster en Psicología de la Educación (a partir de ahora en Psicología Educativa) es que el alumnado adquiera las capacidades y habilidades necesarias para aplicar en este ámbito profesional los conocimientos psicológicos con objeto de promover y optimizar el desarrollo de los individuos y los contextos educativos. 
De acuerdo con la Conferencia de Decanos de Psicología de las Universidades Españolas, los conocimientos básicos más importantes para este perfil son los procesos y etapas del desarrollo normal y anormal y los métodos de evaluación, diagnóstico y tratamientos psicológicos, seguidos de las leyes básicas de los procesos psicológicos y los fundamentos biológicos de la conducta. En esta propuesta formativa, los contenidos de aprendizaje han de guardar una estrecha relación con la competencia profesional vinculada con "el saber hacer algo" a partir de unos conocimientos y con el desempeño de las funciones que le son propias.

Previamente debemos recordar que el marco europeo de cualificaciones incorpora los descriptores del Marco de Cualificaciones de la Educación Superior. El principio de la construcción de estos descriptores o indicadores de nivel son los "learning outcomes". Hoy día los "resultados del aprendizaje" han ido ganando terreno como medio para describir cualificaciones y según Adam (2008), de un instrumento periférico ha pasado a ser el dispositivo radical de la Educación Superior.

El Marco Europeo de las Cualificaciones (EQF) adopta la definición de "learning outcomes" como enunciados de lo que una persona conoce, comprende y es capaz de hacer al término de un proceso de aprendizaje, desglosados en términos de conocimientos, destrezas/capacidades (skills) y competencias, que se pueden adquirir en diversos contextos de aprendizaje (Diario Oficial de la Unión Europea, 2008). La elaboración de esta trilogía descansa en estudios desarrollados por Winterton (2004) y Coles/Oates (2004) y reelaborados dentro del Grupo de Trabajo europeo del EQF (2004/2008). De todos ellos, la competencia se ha convertido en el concepto cardinal de los niveles de referencia del EQF. Por otro lado, la definición e interpretación de términos como conocimientos, aptitudes y competencias se deja en manos de los Estados miembros, y con ello se corre el riesgo de la merma de la transparencia y menoscabo de la función de control de calidad que debería desempeñar este instrumento.
El concepto de competencia cambia según el modelo que se emplee, como se pone de manifiesto en el caso del modelo funcional-conductista en Reino Unido o los modelos multidimensionales de Holanda, Francia o Alemania (Winterton et al. 2005). En el modelo inglés, la competencia se relaciona no con la capacidad global del individuo sino con un conjunto de competencias que son acumulativas antes que integradoras. En el Marco de Cualificaciones alemán el concepto central es el de competencia de acción (Handlungskompetenz) en el contexto de la realización o desempeño al hacer. Los resultados del aprendizaje se describen como grupos de competencias (profesionales, sociales, personales) formuladas en términos de "ser capaz, poder, estar en posesión” (DQR 2010).

Analicemos brevemente otras definiciones de competencia desde el ámbito profesional y educativo. Competencia según Le Boterf (2001), es “la capacidad de movilizar y aplicar correctamente en un entorno laboral determinado, recursos propios (habilidades, conocimientos y actitudes) y recursos del entorno para producir un resultado definido". Nos sentimos más próximos a autores como Echevarría (2001) para quien "posee competencia profesional quien dispone de los conocimientos, destrezas y actitudes necesarias para ejercer su propia actividad laboral, resuelve los problemas de forma autónoma y creativa y está capacitado para colaborar en su entorno laboral y en la organización del trabajo". Según este autor la competencia de acción profesional se compone de cuatro saberes básicos: saber técnico, saber metodológico o saber hacer, saber estar y participar y saber personal o saber ser. En consecuencia, la competencia profesional según Echeverría (2005) incluye:

- Los conocimientos especializados que permiten dominar como experto los contenidos y tareas propias de cada ámbito profesional.

- El saber aplicar los conocimientos a situaciones laborales concretas, utilizando procedimientos adecuados, solucionando problemas de forma autónoma y transfiriendo las experiencias a situaciones novedosas. 
- El estar predispuesto al entendimiento, la comunicación y la cooperación con los demás.

- Y, finalmente, tener un autoconcepto ajustado, seguir las propias convicciones, asumir responsabilidades y tomar decisiones.

En esta misma línea, el término competencias profesionales las aplicamos al psicólogo educativo que interviene en todos los procesos psicológicos que afectan al desarrollo normal y anormal y al aprendizaje, en el marco de los sistemas socioeducativos reglados, no reglados, formales e informales y a lo largo de todo el ciclo vital. Las competencias, en fin, proporcionan una descripción de los distintos roles que desempeña el psicólogo educativo relacionado con las principales áreas de intervención: atención a la diversidad, prevención, desarrollo, proceso de enseñanza aprendizaje y orientación y asesoramiento personal, académico y profesional. En definitiva, se espera que desde esta propuesta el alumnado que curse el máster profesionalizante de Psicología Educativa, pueda adquirir al término del mismo las siguientes competencias genéricas y específicas:

\section{Competencias genéricas:}

- Capacidades para conocer la naturaleza y el marco teórico explicativos de las dificultades, discapacidades y trastornos psicológicos, sociales y educativos.

- Capacidades para aplicar las principales teorías respecto a los procesos psicológicos implicados en las discapacidades, trastornos psicológicos y del aprendizaje.

- Capacidades para conocer los distintos modelos de evaluación e intervención en el ámbito de la Psicología Educativa.

- Capacidades para detectar, comprender y atender las demandas psicológicas de la comunidad educativa, instituciones y servicios comunitarios.

- Capacidades para asesorar, asistir y dar consejo a individuos, grupos o instituciones, a partir de los conocimientos especializados que como orientador se posee.

- Capacidades para mediar entre dos o más contextos, instituciones, grupos o personas, con el fin de llegar a acuerdos.

- Capacidades personales e interpersonales para el trabajo en equipo.

- Capacidades para diseñar, planificar y seleccionar técnicas y recursos de intervención e investigación.

- Capacidades comunicativas, de escucha, creatividad, flexibilidad y persuasión.

- Capacidades para conocer y ajustarse al código deontológico de la Psicología Educativa.

\section{Competencias específicas:}

- Capacidades para orientar a las personas a lo largo del ciclo vital, en el aspecto psicológico, educativo, personal y profesional

- Capacidades para identificar factores personales, psicológicos, sociales y educativos que favorecen o dificultan el proceso de aprendizaje.

- Capacidades para intervenir en los distintos contextos: escolar, familiar, medios comunitarios, organizaciones, etc.

- Capacidades para seleccionar y aplicar las principales técnicas, estrategias y programas de intervención psicoeducativa.

- Capacidades para definir objetivos, trazar el plan de intervención, realizar el seguimiento y evaluación de la propuesta de actuación psicológica.

- Capacidades para mediar en la modificación de aquellas circunstancias del contexto generadoras de problemas, así como dotar a los individuos de las adecuadas competencias para que puedan afrontar con éxito las situaciones problemáticas.

- Capacidades para organizar, enfocar, seleccionar, filtrar o determinar el orden de aparición de los hechos, para lograr el consenso, el cambio o la transformación. 
- Capacidades para intervenir desde un enfoque sistémico o global desde el que se aborda no sólo al sujeto sino también el contexto sociofamiliar y escolar.

- Capacidades para colaborar en el proceso de ayuda para promover el desarrollo integral del potencial de cada persona.

- Capacidades para conocer las bases para la selección de los métodos y técnicas de evaluación adecuados a cada situación o contexto.

- Capacidades para seleccionar y aplicar pruebas e instrumentos de evaluación psicológica individuales y grupales.

- Capacidades para analizar e interpretar los resultados de una evaluación psicológica.

- Capacidades para diagnosticar, elaborar un informe u orientar una propuesta de intervención.

- Capacidades para recoger, analizar y valorar información relevante, sobre los diversos elementos que intervienen en el proceso de enseñanza/aprendizaje.

- Capacidades para gestionar y promover la coordinación con otros profesionales, instituciones y servicios.

- Capacidades para responder a las demandas de las instituciones y de la Administración educativa relativas a informes y dictámenes, especialmente aquellos referidos a los alumnos con necesidades educativas especiales.

\section{Estructura y contenidos}

Dado que a nivel normativo no se regula detalladamente, los contenidos de los programas de postgrado, atendiendo a la diversidad de universidades españolas y al ámbito de su autonomía, consideramos que desde la Conferencia de Decanos de Psicología y en estrecha coordinación con el Consejo General de Colegios Oficiales de Psicólogos, se debe impulsar políticas encaminadas a que la programación de las enseñanzas se correspondan, como indica el propio MEC, con su ámbito científico, tecnológico o profesional y que la denominación del título sea acorde con su contenido y no conduzca a error sobre su nivel o efectos académicos ni a confusión sobre su contenido y, en su caso, efectos profesionales. Hoy día, con el título de Máster en Psicología Educativa, encontramos una oferta muy amplia y diversa en cuanto a contenidos, que lamentablemente tienen en común la ausencia de criterios de acceso específicos para la selección del alumnado, lo que permite que cualquier licenciado o graduado, curse esta formación especializada a pesar de que su formación inicial, básica y fundamental no se corresponda con el ámbito científico o profesional de las especialidades de Psicología.

En concreto, sería conveniente que las distintas facultades de Psicología en las que se imparta el Máster de Psicología de la Educación, llegaran a un consenso en la estructura modular de programa, al menos en los contenidos transversales y comunes. De cualquier modo, la formación especializada que reciba el psicólogo educativo debe permitirle desarrollar las competencias necesarias para responder a las siguientes demandas:

- En el marco de la atención a la diversidad. Su intervención es fundamental en la detección, diagnóstico, orientación y seguimiento del alumnado con necesidades educativas especificas, con independencia de las causas que las determinen (marginalidad, sobredotación, discapacidad, minorías étnicas, desmotivación, etc.) y lo que es más importante, intervenir en la modificación de los elementos del entorno o del proceso de enseñanza-aprendizaje que puedan generar las dificultades de aprendizaje, para en la medida de lo posible, anticiparse y promover acciones encaminadas a evitar su aparición y reducir sus efectos. La intervención orientadora en el ámbito de la atención a la diversidad, no se circunscribe exclusivamente al contexto escolar, sino que se extiende al sociofamiliar, al clínico y al laboral.

- En los procesos de enseñanza-aprendizaje. Facilitando las estrategias de aprendizaje autónomo más apropiadas en cada situación, en fun- 
ción de las características personales del alumnado. Estrategias tales como habilidades y técnicas de estudio, aprender a aprender y aprender a pensar, técnicas motivacionales, habilidades de concentración y comunicativas, resolución de problemas, etc.

- En el asesoramiento y la orientación profesional. El rol del psicólogo será el de educar, formar y asesorar, aportando estrategias y presentando propuestas que ayuden a alcanzar la madurez personal, educativa y vocacional que necesita el alumnado para la toma de decisiones. El psicólogo actúa como mediador entre la persona y el medio sociolaboral En la actualidad, uno de los aspectos prioritarios, además de la información-formación profesional, es el proceso de toma de decisiones. En este proceso, se toman en consideración intereses, aptitudes, personalidad y otras características sociales y personales, para confrontarlas con opciones de estudio y la elección de una profesión. Las habilidades en la toma de decisiones, una vez que se adquieren se aplican, además de a las decisiones profesionales, a diversas situaciones de la vida cotidiana.

- En el proceso de ayuda para promover el desarrollo integral, a partir del potencial de cada persona. El psicólogo intervendrá diseñando y coordinando programas para promover el desarrollo del auto-concepto, la autoestima y el desarrollo de habilidades sociales y de comunicación y dotando a la persona de las competencias necesarias para afrontar las demandas de cada etapa evolutiva y proporcionándole las estrategias y habilidades para la vida que le permitan la utilización de comportamientos adecuados para la resolución de problemas relacionados con asuntos familiares, personales, de tiempo libre, de trabajo o de la comunidad.

- Orientación para la prevención, encaminada a anticiparse a la aparición de elementos que obstaculicen el desarrollo y el proceso de enseñanza-aprendizaje de los alumnos. Aspectos esenciales a prevenir serían: el fracaso escolar y pro- fesional, la violencia, estrés, tabaquismo, alcoholismo y consumo de drogas, sida y embarazos no deseados, etc.

- Asesoramiento sociofamiliar, educativo e institucional. El psicólogo actúa como mediador entre la familia y la escuela, procurando la información y el apoyo técnico a profesores y padres e impulsando la cooperación de los servicios, instituciones y organizaciones sociales del entorno

Llegados a este punto, ¿qué formación de postgrado requeriría un psicólogo educativo? Compartimos los acuerdos a que en su día llegaron la Conferencia de Decanos de Psicología en cuanto a la duración, categorías de contenidos y los criterios específicos de acceso. Es decir: duración de 90 créditos, distribuidos en los distintos contenidos transversales, comunes, complementarios, prácticas profesionales supervisadas y proyecto final de máster. Del mismo modo se requerirá al alumnado, tanto español como extranjero, el haber cursado el grado o la licenciatura en Psicología para poder acceder a esta formación y profesión.

Como indicamos al inicio de este trabajo, en él sólo haremos referencia a un tipo de máster, aquel que capacita al psicólogo para la práctica profesional en contextos educativos y que da acceso a una profesión regulada, dentro de la especialidad de Psicología y Pedagogía (hoy denominada Orientación Educativa) de Educación Secundaria. Evidentemente, no compartimos que a esta especialidad pueda acceder cualquier docente que curse el citado Máster de Formación del Profesorado.

Asimismo, se propone un programa de postgrado inter-universitario con la participación de las universidades españolas y universidades extrajeras. El Ministerio de Educación y Ciencia regulará las condiciones y requisitos para la homologación del título y la organización de los estudios que incluirán el establecimiento de convenios entre las universidades participantes. En el convenio se establecerán las condiciones para el desarrollo del programa y para la gestión de los expedientes de los estudiantes, así como los procedimientos para la expedición y custodia del título. 
Previo a la presentación de la estructura del máster propuesto, describamos brevemente las distintas categorías de contenidos que integra:

- Contenidos Transversales: aquellos que serán comunes a los distintos másteres que integran el Programa Oficial de Postgrado (POP) en Psicología y que constará de 10 créditos $(9 \%)$. Las Materias Transversales, como indica la Conferencia de Decanos, tienen como objetivo, el profundizar y lograr la integración de los contenidos académicos y las competencias adquiridas en el Grado o la Licenciatura. Así como, el manejo de la información científica, el uso de las nuevas tecnologías, el desarrollo de competencias de investigación y el diseño de programas, estrategias e instrumentos de evaluación, etc.

- Contenidos Comunes: que integrarán las materias troncales del Máster y que guardan una estrecha relación con las principales demandas que desde la Comunidad Educativa, instituciones y servicios comunitarios se les solicitan. La asignación porcentual de créditos a estos contenidos es del 35\%. Estas materias deberán ser cursadas por todo el alumnado para la adquisición de las competencias profesionales específicas asociadas al Máster de Psicología de la Educación.

- Contenidos Complementarios: con los que se garantizará la optatividad dentro del propio Máster y una mayor especialización relacionadas con las funciones de sector y los tipos de centros y del alumnado que lo integra. Asimismo, ayudará al alumnado a ir perfilando su formación de acuerdo a sus intereses para trabajar en sistemas externos o internos de apoyo psicoeducativo. La asignación porcentual de créditos a estos contenidos es del $30 \%$.

- Prácticas Profesionales Supervisadas: se consideran de vital importancia y dada la diversidad de discapacidades, trastornos y problemáticas, propias de este contexto. Se recomendará que al menos el alumnado curse las prácticas en dos modalidades distintas en relación a la edad o tipo de problemática que presente el alumnado. En consecuencia, las prácticas se realizarán bajo la tutela de otros psicólogos educativos acreditados que realicen su labor en centros específicos, ordinarios con apoyo, de alumnado de alto riesgo, adultos, centros educativos terapéuticos, fundaciones e instituciones reconocidas mediante convenio con las Universidades.

- Proyecto Final de Máster: Trabajo que al final del ciclo formativo deberá presentar y defender ante un tribunal compuesto por el profesorado que imparte el Máster. Se pretende que el título de máster proporcione las capacidades adicionales a las que otorgan los títulos de de Licenciatura o de Grado. La asignación porcentual de créditos para las prácticas supervisadas y el proyecto final de máster es del $35 \%$.

A continuación, presentamos en la tabla 1, la estructura del Máster de Psicología Educativa (de 90 créditos), al que nos hemos referido a lo largo de este artículo, sabiendo que las materias o asignaturas propuestas no cubren por completo y en su totalidad este ámbito, pero entendemos que es posible la complementariedad desde el trabajo en equipo de los profesionales que formen parte de estos servicios psicoeducativos.

\section{Consideraciones finales}

En el ámbito universitario, el actual curso académico 2010-2011 es de especial significación pues durante el mismo, se pretende alcanzar la completa reforma curricular en el marco del Espacio Europeo de Educación Superior. Las acciones previstas se encuadran en la "Estrategia Universidad 2015", para la modernización del sistema universitario español, fomentando la transparencia y promoviendo la competencia entre las distintas instituciones. La finalidad última es convertir a las universidades en el motor de la sociedad basada en el conocimiento, prestando una especial atención a la racionalización de los másteres y los doctorados. 
Tabla 1. Estructura curricular del Máster de Psicología Eductiva

BLOQUES

CONTENIDOS

Evaluación y diagnóstico

Psicoeducativo

Contenidos

Comunes

$(35 \%)$

Psicología de la Educación Especial

Intervención, Orientación
y Asesoramiento Psicológico

Atención a la diversidad e intercultu-

ralidad

Contenidos

Complementarios

(30\%)
Intervención psicológica: para la prevención y el desarrollo

\section{MATERIAS}

1. Concepto y objeto de la evaluación en las distintas edades y contextos.

2. Procedimientos, técnicas e instrumentos de evaluación.

3. Evaluación diagnóstica, elaboración de informes, dictámenes y documentos de derivación a distintas instituciones.

4. Evaluación de programas, de la enseñanza, de la organización escolar, etc.

1. Discapacidades cognitivas y comunicativas (discapacidad intelectual, trastornos del espectro autista, disfasias, etc.)

2. Discapacidades sensoriales y físicas (motóricos, hipoacúsicos y sordos, ciegos y amblíopes).

3. Discapacidades comportamentales.

4. Alumnos de altas capacidades.

1. Contexto familiar y escolar.

2. Contexto social y comunitario.

3. Institucional y de servicios

1. Compensación educativa.

2. Grupos de riesgo.

3. Minorías étnicas y marginalidad.

1. Prevención del fracaso escolar, la violencia, la drogadicción, etc.

2. Desarrollo del autoconcepto, asertividad, habilidades sociales y comunicativas, etc.

Dificultades de aprendizaje y problemas de comportamiento

1. Trastornos del lenguaje lector, escritor y numérico.

2. Déficit de atención.

3. Conductas disruptivas, desafiantes, indisciplina, etc

\begin{tabular}{|c|c|c|}
\hline $\begin{array}{l}\text { Prácticas } \\
\text { Profesionales }\end{array}$ & $\begin{array}{l}\text { Prácticas profesionales } \\
\text { supervisadas por profesionales titula- } \\
\text { dos }\end{array}$ & $\begin{array}{l}\text { 1. Centros de Educación Especial. } \\
\text { 2. Centros ordinarios con apoyo, de infantil, primaria y secundaria. } \\
\text { 3. Centros de adultos y de Formación Profesional. } \\
\text { 4. Aulas hospitalarias y Centros Educativos Terapéuticos, etc. }\end{array}$ \\
\hline $\begin{array}{l}\text { Proyecto Final de Máster } \\
(35 \%)\end{array}$ & Proyecto Final de Máster & $\begin{array}{l}\text { 1. Investigación aplicada al ámbito de la Psicología Educativa. } \\
\text { 2. Evaluación, intervención y seguimiento de un supuesto práctico. } \\
\text { 3. Diseño, realización y valoración de un programa de intervención } \\
\text { realizado en centros educativos. }\end{array}$ \\
\hline
\end{tabular}

Contenidos

Transversales

(10 créditos)

1. Uso de las nuevas tecnologías de la información y comunicación en contextos socioeducativos.

2. Métodos, estrategias y técnicas metodológicas aplicados en distintos contextos y situaciones.

3. Métodos y procesos de búsqueda, organización y comunicación de la información.

Existen dudas razonables sobre que en el presente curso se alcance la completa reforma curricular, máxime cuando en España llevamos un considerable retraso en procesos previos. Efectivamente, como recoge el reciente documento The development of national qualifications frameworks in Europe (Cedefop, 2010), la mayoría de los países europeos, se encuentran en un proceso de desarrollo o de implementación de los marcos nacionales de cualificaciones en el marco europeo de cualificaciones. De España se indica que no existe información al respecto.

Pero lo más preocupante no es lo que queda por hacer, sino parte de lo que ya se ha hecho. Nos referimos al Máster de Formación del Profesorado, en la especialidad de "Orientación Educativa" que permite el acceso a actividades profesionales reguladas a cualquier docente, con independencia de su formación básica, específica y fundamental. Evidentemente, esta 
nueva propuesta en la reforma de los estudios universitarios tiene serias implicaciones para la profesión del psicólogo en nuestro país. Quizás el problema radique en una concepción no fundamentada científicamente de la Orientación Educativa. Más que una "ciencia de la acción” y "una profesión", la Administración Educativa parece entenderla como una tarea que puede realizar cualquier graduado o licenciado que haya cursado el citado Máster.

En España, a pesar de los avances científicos producidos en los últimos años, la Orientación Educativa ha sufrido un lamentable retroceso, ocasionado primero por la Especialidad de Psicopedagogía y ahora por el acceso a la profesión de cualquier graduado, a través del Máster de Formación del Profesorado. En nuestro país, para ejercer la medicina se necesita ser médico, para la venta de medicamentos, ser un titular de farmacia; por el contrario, la evaluación diagnóstica y el asesoramiento psicológico del alumnado con discapacidad intelectual, sensorial o motora, conductas asociales, trastornos del espectro autista, de personalidad o de conducta, en definitiva la salud mental, según la Administración Educativa, la puede hacer cualquier docente.

Ante esta situación, cada vez cobra más fuerza el reciente acuerdo firmado en septiembre de 2010, por la Conferencia de Decanos de Psicología (CDPUE), Consejo General de Colegios Oficiales de Psicólogos, la Federación de Asociaciones de Directivos de Centros Educativos Públicos (FEDADI), la Confederación Española de Asociaciones de Madres y Padres de Alumnos (CEAPA) y la Confederación Católica Nacional de Padres de Familia y Padres de Alumnos (CONCAPA), porque es evidente que en una escuela, tan compleja como la actual, no cabe una intervención eficiente desde la pseudo-profesionalización, dado que desde ella, no sólo se va en contra de la calidad educativa, sino que además constituye un fraude y un daño irreparable al usuario.

\section{Referencias}

Adam, S. (2008). Learning outcomes, current developments in Europe: Update on the issues and applications of learning outcomes associated with the Bologna process. Contribution to the Bologna Seminar: Learning outcomes based higher education: the Scottish experience. 21-22 February 2008, at Heriot-Watt University, Edinburgh, Scotland.

Cedefop (2009). The shift to learning outcomes. Policies and practices in Europe. Luxembourg: Office for Official Publications of the European Communities.

Cedefop (2010). The development of national qualifications frameworks in Europe. Luxembourg: Office for Official Publications of the European Communities.

Coles, M. y Oates, T. (2004). European reference levels for education and training. An important parameter for promoting credit transfer and mutual trust. (Study commissioned to the QCA London). Luxemburgo: Oficina de Publicaciones Oficiales de las Comunidades Europeas, 2004. (Cedefop Panorama series;109).

Diario Oficial de la Unión Europea (2005). Directiva 205/36/CE del Parlamento Europeo y del Consejo de 7 de septiembre de 2005 relativa al reconocimiento de cualificaciones profesionales. (Texto pertinente a efectos del EEE), 2005, L255/22.

Diario Oficial de la Unión Europea (2008). Recomendación del Parlamento Europeo y del Consejo de 23 de abril de 2008 relativa a la creación del Marco Europeo de Cualificaciones para el aprendizaje permanente. (Texto pertinente a efectos del EEE), 2008, C 111/1-7.

DQR (2010). Expertenvotum zur zweiten Erarbeitungsphase des Deutschen Qualifikationsrahmens. Einführung. DQR-Büro. Juli 2010. Berlin.

Echeverría, B. (2001). Configuración actual de la profesionalidad. Letras de Deusto, 31, 35-55.

Echeverría, B. (2005). Competencias de acción de los profesionales de la orientación. Madrid: ESIC editorial.

European Commission (2005). Towards a European Qualifications Framework for Lifelong Learning. 
Commission Staff Working Document. Brussels.

Le Boterf, G. (2001). Ingeniería de las competencias. Barcelona: Gedisa.

Pérez Solís, M. (2010). ¿Puede un docente sustituir en su rol profesional al psicólogo educativo? Papeles del Psicólogo, 31, 143-154.
Winterton, J., Delamare-Le Deist, F. y Stringfellow, E. (2006). Typology of knowledge, skills and competences: Clarification of the concept and prototype. Luxemburgo: Oficina de Publicaciones Ofi-ciales de las Comunidades Europeas, (Cedefop Reference series).

Manuscrito recibido: 05/11/2010

Revisión recibida: 28/12/2010 Manuscrito aceptado: 03/01/2011 